\title{
OS TERRITORIOS DE
}
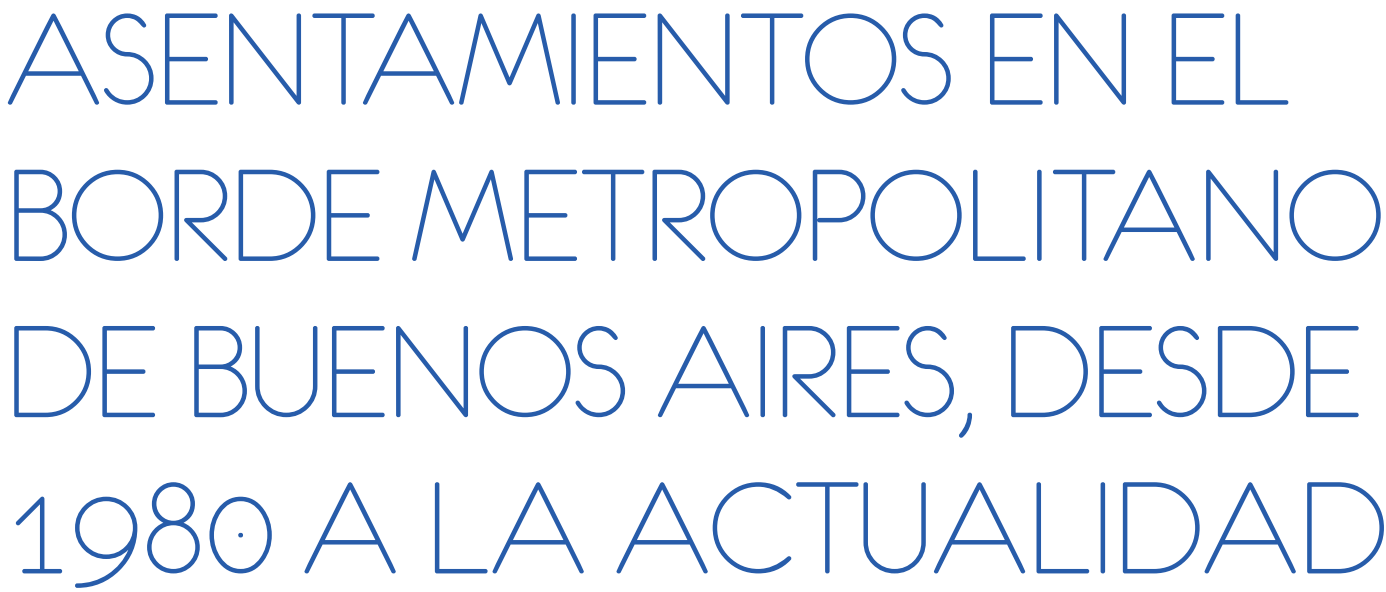

\section{THE SETTLEMENT TERRITORIES ON THE METROPOLITAN FRINGES OF BUENOS AIRES,}

FROM 1980 TO THE PRESENT DAY

LUCAS JORDÁN DOMBROSKI 1

1 El artículo fue desarrollado en el marco del proyecto "Instrumentos de planificación y gestión para el Área Metropolitana de Buenos Aires. Actores, políticas públicas y configuraciones territoriales PIO-CONICET-UNGS" (PIO CONICET UNGS-144-20140100020-CO) dirigido por la Dra. Alicia Novick.

2 Licenciado Urbanista

Consejo Nacional de Investigaciones Científicas y Tecnicas de Argentina (CONCET)

Becario doctoral del Consejo en Estudios Urbanos, Doctorando en Estudios Urbanos,

Instituto del Conurbano, Universidad Nacional de General Sarmiento

https://orcid.org/0000-0002-3940-1632

jordan.dombroski@gmail.com 
Los "asentamientos" o "tomas de tierra" en el Gran Buenos Aires han sido objeto de estudios y debate desde fines de los años 80. Las características de las áreas ocupadas, las localizaciones y quienes intervienen desde diferentes lugares en el proceso, han sido parte importante de las cuestiones sobre la que los especialistas han trabajado. No obstante, algunas relaciones entre esas especificidades han sido poco exploradas. En ese contexto, el objetivo de este trabajo es reconstruir las lecturas del proceso de tomas de tierras en la metrópoli desde 1980 a la actualidad, en torno a los actores involucrados y a las especificidades del suelo, a fin de comprender los territorios en cuestión. Para esto se recurrió a diferentes textos y fuentes primarias. Como resultado, se advirtió que a lo largo del tiempo se han ido definiendo momentos muy específicos con participación, protagonismo y construcción de diferentes actores según las coyunturas, y que ello guarda estrecha relación con la concentración o dispersión de las tomas en áreas bien definidas.

Palabras clave: asentamientos, tomas de tierra, territorios, localización, actores, Gran Buenos Aires.

The "settlements" or "land occupations" in Greater Buenos Aires have been the subject of studies and debate since the late 1980s. The characteristics of the occupied areas, the locations and who intervenes in the process from different places have been an important part of the questions the specialists have worked on. However, some relationships between these specificities have barely been researched. The goal of this work is to reconstruct the interpretations of the land occupation process in the metropolis from 1980 to the present day, around the players involved and the specificities of the land; for the purposes of understanding the territories in question. For this, different texts and primary sources were used. As a result, it will be seen that, over time, very specific moments were defined with participation, prominence and construction of different players based on the circumstances, and that this is closely related to the concentration or spread of occupations in well-defined areas

Keywords: Settlements, Land Occupations, Territories, Location, Players, Greater Buenos Aires. 


\section{INTRODUCCIÓN}

El hábitat informal es un tema relevante en América Latina que, sobre todo, desde los años 1970 ha suscitado una amplia gama de debates que intentaron dar respuestas al fenómeno en una escala regional (Turner, 1977; Pradilla, 1982; Hardoy y Satterthwaite, 1987; Clichevsky, 2003). Más recientemente, los estudios se han orientado al análisis de casos desde donde se intenta comprender las especificidades del fenómeno para cada sitio, pues la problemática es substancialmente diferente en las grandes ciudades del subcontinente. Mientras que para ciudad de México, San Pablo, Río de Janeiro, Lima, Caracas o Bogotá la informalidad representa entre el 30 y 60 por ciento, en otras como Santiago o Montevideo, representa en torno al 10 por ciento (Clichevsky, 1991), al igual que para el Gran Buenos Aires 3 (GBA), donde existe un millón y medio de habitantes viviendo en más de mil barrios (Cravino, 2018). La especificidad de los procesos también se manifiesta en términos ambientales, sociales y políticos. En el GBA, los "asentamientos" $\mathbf{4}$ o "tomas de tierra" han sido objeto de estudios académicos, notas de prensa, debates en las gestiones de gobiernos y políticas, desde fines del siglo pasado, cuando se evidenció que se trataba de un fenómeno que había llegado para quedarse, y que resultaba muy diferente a las denominadas "villas" y otros procesos de hábitat informal. La localización de ellos y los actores involucrados aquí han sido igualmente tema de relevancia y objeto de diversos estudios.

A diferencia de las villas, que surgieron alrededor de la década de 1930 y se concentran en la Ciudad de Buenos Aires y los municipios del primer cordón, los asentamientos iniciaron a principios de los 80 y se han ido construyendo en el borde del área urbanizada. Sobre este fenómeno, la bibliografía especializada (Izaguirre y Aristizabal, 1988; Merklen, 1997; Di Virgilio, Arqueros y Guevara, 2012; Cravino, 2018) considera como una cuestión importante su localización "en municipios de la segunda corona" (Di Virgilio et al., 2012, p. 32), en general, en áreas no consolidadas, "en su inmensa mayoría ubicados sobre tierra privada, (...) en terrenos que por lo general eran basurales, pajonales, o inundables, por lo que los dueños no tenían un interés o posibilidad en explotarlo económicamente o sufrían restricciones normativas para esto" (Cravino 2001, p. 8). Asimismo, a lo largo del tiempo las organizaciones políticas, comunitarias, vecinos organizados, funcionarios públicos y otros actores que han participado de las tomas fueron definidos como diferentes a los de las villas, y han ido cambiando a lo largo del tiempo y construyéndose como tales en torno al proceso de asentamientos.
Como se verá a lo largo del artículo, muchos trabajos explican las razones de surgimiento y extensión de las tomas, los actores que participan, las especificidades físicas de los barrios o su localización. No obstante, algunas relaciones entre estas especificidades dadas a través del tiempo no han sido suficientemente exploradas. En tal sentido, el objetivo del presente trabajo es reconstruir las lecturas del proceso de tomas de tierras en el GBA desde 1980 a la actualidad, en torno a los actores que participan y las especificidades del suelo ocupado; con el fin de comprender, los territorios de los que se trata y sus determinaciones en lo metropolitano. Esto implica consultar diferentes materiales, desde textos de especialistas a fuentes primarias de tipo cualitativas, así como planos y datos cuantitativos. Desde la óptica de la acción pública, con el análisis del accionar de múltiples actores que se articulan en torno al proceso de extensión de los asentamientos a lo largo del tiempo, se entiende que esta lectura pone de manifiesto las particularidades materiales y sociales, y resulta fundamental para discernir el proceso y diseñar políticas públicas más eficaces.

\section{MARCO TEÓRICO}

El presente trabajo se nutre de diferentes aportes disciplinares y perspectivas de análisis en relación al estudio de los actores y el territorio. Desde ese lugar, y en cuanto a los trabajos que abordan las políticas públicas, se considera que es preciso desarmar la idea de Estado, que se asume como uno monolítico, en una dirección de tipo jerárquica, racional y estática de las políticas (Ugalde, 2014) que se desarrollan en torno al hábitat informal; y pensar, más bien, la interacción de los actores en términos de autoridades públicas y actores sociales, pues la implementación y la redefinición de las políticas de hábitat se juega en el territorio, donde los actores además se construyen como tales desde su participación (Chiara y Catenazzi, 2009). Esto último remite a hablar de acción pública, ya no solo en relación a las políticas en su elaboración e implementación, sino en un ámbito más extenso que conlleva considerar las transformaciones urbanas en general y que permite visualizar con mayor precisión el accionar de las autoridades públicas, como también concebir de manera diferente a los actores sociales en su capacidad de incidencia sobre dichas transformaciones.

Ahora bien, ¿qué se entiende por "acción pública"? En términos teóricos, y para el estudio de los asentamientos, conviene entender el concepto como lo plantea Thoenig (1997, p. 30), donde el poder público no tiene el monopolio de lo político

3 Aquí se considera el Gran Buenos Aires comprendido por la Ciudad Autónoma y los 30 municipios que la rodean, los cuales conforman tres anillos o coronas sucesivos, desde el centro a la periferia.

4 Existe acuerdo entre los investigadores especializados en el estudio del hábitat informal en el GBA en cuanto a la denominación de "tomas de tierra" y "asentamientos (a secas)" como equivalentes. La denominación "asentamientos informales" o "asentamientos populares" refiere a un proceso más general que incluye, por ejemplo, a las "villas". 
y las transformaciones, en este caso la extensión de los asentamientos, "se da(n) en una "arena" en la cual coexisten diferentes lógicas y valores, modalidades muy diversas, coyunturas e intervenciones planificadas, racionalidad técnica y elecciones políticas, expertises científicas y compromisos militantes, programación y concertación". Esto supone comprender el estudio de los actores y las transformaciones territoriales desde esa "arena", que algunos autores operativizan en torno a la identificación de distintos "momentos" (Merlinsky, 2017) de interacción o puntos de inflexión en un proceso.

Las consideraciones del territorio se juegan en un plano diferente, pero que aquí interactúan con la de acción pública como noción. Así, se entiende al territorio como un "ámbito dinámico y activo, una estructura social que deriva de la interacción de los sujetos y las características específicas y físicas materiales e inmateriales de los diferentes espacios" (Governa, 2007, p. 23), por lo que, además de tratarse de un área específica compuesta por extensiones, límites y obstáculos, consta de flujos y nodos que comportan redes de relacionamiento y conexiones de los actores (Amin, 2005). A partir de ello, este trabajo se centra en algunas de las especificidades territoriales que pueden resultar de interés desde una escala metropolitana.

\section{METODOLOCÍA}

Los territorios de asentamientos, como objeto de estudio, y en los términos que aquí interesan constan de los actores que participan -desde la óptica de la acción pública-y las especificidades de los lugares en donde se sitúan los barrios, tanto espacial como temporalmente.

La técnica de estudio consistió en una exploración en clave histórica acerca de las lecturas de proceso de tomas de tierra producidas entre los años 1980 y principios del presente siglo. Para los años más recientes, la técnica se basó en la construcción de cartografía a escala metropolitana con la ubicación geográfica y temporal de los barrios, y la identificación de los diferentes actores involucrados.

Se recurrió a los textos de especialistas, prestando especial atención a las valoraciones de los autores sobre las características de las áreas ocupadas, la localización de las tomas, los actores participantes, sus modos organizativos y vinculaciones con otros actores. Con el propósito de reconstruir los procesos más recientes, se recurrió a datos de plataformas Web (Registro Provincial de Villas y Asentamientos por Ley de Acceso Justo al Hábitat 14.449, Registro Nacional de Barrios Populares y Google Earth). Además, en esta instancia, en un nivel de aproximación diferente, el estudio de casos producto de las exploraciones de tesis doctoral que elabora el autor resultó relevante, desde el aporte de información sobre los territorios tanto en sus especificidades materiales como inmateriales. Las entrevistas semiestructuradas a actores clave (un total de 15 realizadas a funcionarios municipales, referentes vecinales y miembros de organizaciones), la elaboración de cartografía intencionada, y el trabajo de campo desarrollado desde el estudio de barrios de los municipios que más tomas han tenido (Moreno, José C. Paz y La Matanza), ayudaron a comprender por qué se ocuparon esas áreas y cómo, los actores que participaron, las políticas que se aplicaron y las estrategias y recursos que se desplegaron.

Desde la reconstrucción del proceso de tomas se pudieron identificar "momentos" diferentes en cuanto a la articulación de los actores y las áreas que se ocuparon. Así, se trata de especificidades territoriales que varían en el tiempo, y que en adelante se pondrán de manifiesto.

\section{IV.RESULTADOS}

Los resultados se presentan en dos partes, una primera vinculada al análisis de la bibliografía, en el período que va desde la década de 1980 hasta el año 2003, y otra, que toma desde 2003 en adelante, donde se avanza sobre datos cuantitativos y cualitativos del fenómeno.

\section{La construcción -desde la bibliografía- de los territorios de asentamientos en las últimas décadas}

Antes de abocarse al largo derrotero de los asentamientos hasta la actualidad, es preciso dar cuenta de su origen. Pues, hasta los años 70 existían posibilidades menos restrictivas de acceso al suelo y vivienda para las clases populares, en comparación con las actuales, vinculadas a políticas de Estado y dinámicas de mercado. Sobresale, por su relevancia, el papel de la medida de congelamiento de alquileres y los "loteos baratos" (sin requerimiento de servicios básicos) (Cravino, 2001; Di Virgilio et al., 2012) que tuvieron su fin durante la dictadura cívico-militar, con la sanción de la ley de Locaciones Urbanas № 21342 del '76 y el decreto-ley de Usos del Suelo y Ordenamiento Territorial $N^{\circ}$ 8912 de 1977. Como parte del paquete de medidas, también es necesario señalar la sanción del Código de Planeamiento del municipio de la ciudad de Bs. As. y la expulsión de población por erradicación de las villas (Pacheco, 2018) o por demoliciones para la construcción de autopistas (Izaguirre y Aristizabal, 1988; Cravino, 2001).

Desde el análisis de la bibliografía especializada, se pueden advertir cuatro momentos en el proceso de construcción de los territorios de asentamientos.

\section{Primer momento. Las tomas en la zona Sur y la organización eclesial} Hacia principios de los 80, en el ocaso de la dictadura cívicomilitar los asentamientos surgieron como un nuevo fenómeno en la zona sur del Gran Buenos Aires, sobre todo en ocupaciones de grandes predios vacantes; proceso asociado al accionar de grupos eclesiales que, en gran medida, por la proscripción 


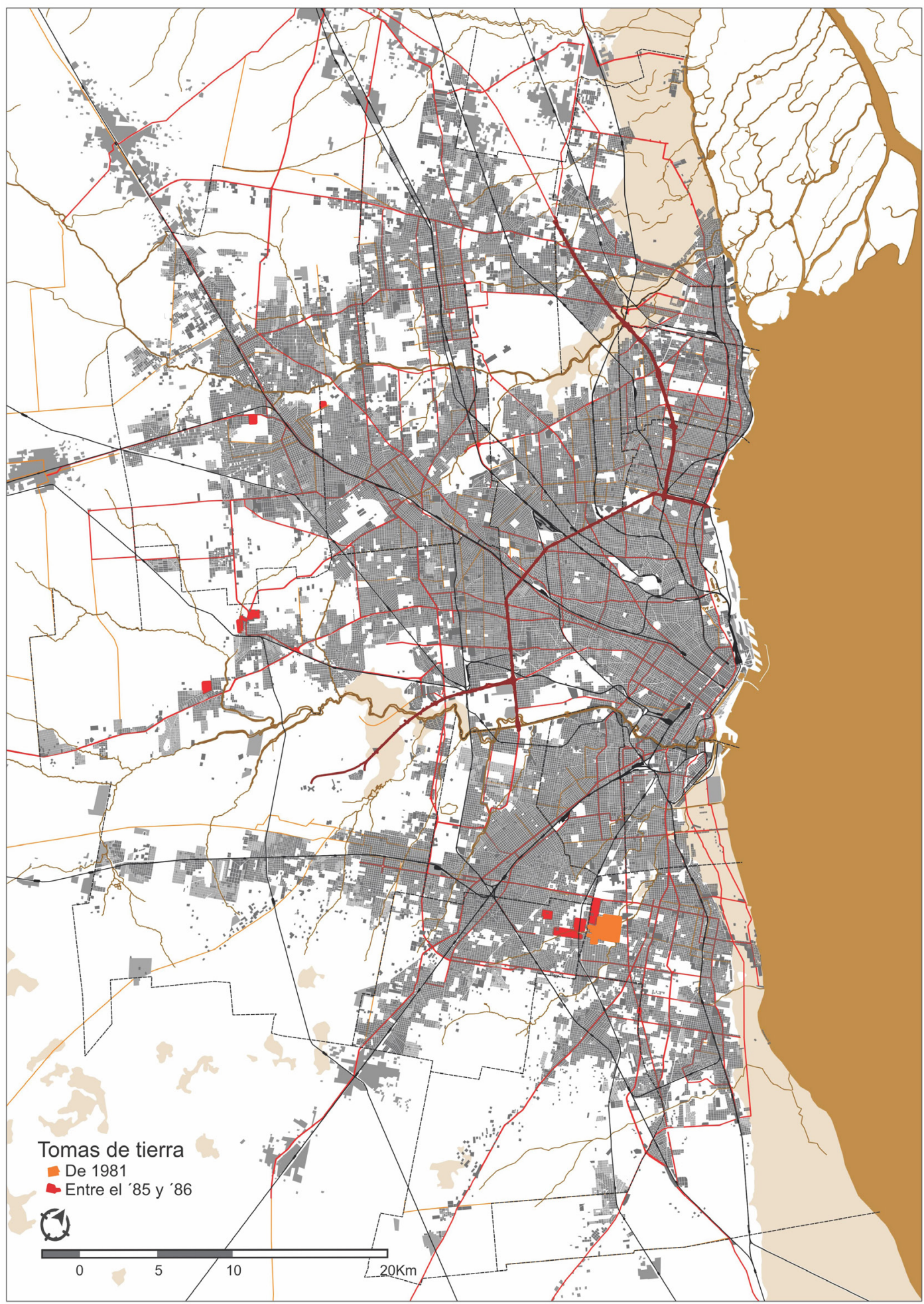

Figura 1. Las tomas de tierra desde 1981 a 1986. Fuente: Elaboración del autor a partir de los registros de Izaguirre y Aristizabal (1988). 
política que imperaba en esos años se habían constituido en actor clave de contención de la población empobrecida y desplazada. La organización eclesial, a lo largo de los años, fue expandiendo su influencia en relación al acompañamiento en las tomas, las cuales se dieron, tal como señala Cravino desde el sur, "hacia el oeste y en menor medida al norte -zona donde se concentran los sectores de altos ingresos-" (2001, p. 10).

Uno de los primeros trabajos que refiere al fenómeno de asentamientos es el de Izaguirre y Aristizabal (1988), que trata las tomas colectivas y organizadas de tierras, considerando el papel de las clases populares y su devenir en el contexto de dictadura y la vuelta a la democracia. Es desde ese lugar que se plantea una vinculación directa entre la generación de grandes obras de infraestructura para el GBA y la localización de las tomas de tierra. De esta manera, la proyección de construcción y conformación parcial del Cinturón Ecológico y la Autopista del Buen Ayre se constituyeron como proyectos que delimitaban las áreas que se pretendían exentas de asentamientos de las que podrían ser susceptibles de nuevas tomas. Dichas autoras entienden que existe, por entonces, un "paquete" de medidas que produjo diferentes efectos en los grupos de población afectada, y señalan al respecto una intencionalidad muy clara: "empujar a los pobres urbanos hacia la periferia de los partidos del Gran Buenos Aires, por detrás del corredor libre denominado "cinturón ecológico", futura área verde de reserva que, bajo el ropaje ideológico del "aire limpio" facilitaba el control estratégico de los sectores más desposeídos..." y, en consecuencia, "dispersar a esos mismos sectores en un radio mucho más extenso, lo que limitaba su amenazante concentración en las proximidades del asiento de los grupos de poder" (Izaguirre y Aristizabal, 1988, p. 7).

El siguiente plano (Figura 1), reconstruido a partir de los datos recopilados por Aristizabal, muestra los asentamientos producto de las tomas del año 1981 y las que se dieron posteriormente, ya en democracia entre 1985 y 1986. Si bien, los registros de entonces no son muy precisos y están basados especialmente en informes periodísticos, se puede apreciar con claridad la importancia de las tomas en el límite entre Almirante Brown y Quilmes, que comparten nueve barrios, en las márgenes de los arroyos Las Piedras y San Francisco, en el área de influencia del Obispado de Quilmes (seis en 1981 y tres en 1985). Más adelante, en el año '86 se registraron tres tomas en La Matanza, una en Merlo y una en Morón, pero todas de menor magnitud en cuanto a superficie ocupada y cantidad de familias.

\section{Segundo momento. Las tomas en algunos municipios de la} segunda corona, desde las organizaciones "a-partidarias" a los vínculos con el peronismo.

En los primeros años de democracia, durante el radicalismo, "el planteo estatal había estado dirigido a tratar de evitar las ocupaciones, confrontando con los movimientos de ocupantes" (Merklen, 1997, p. 7). Desde el gobierno peronista la relación se presentó de manera diferente, construyéndose así zonas muy específicas en donde se desarrollaron asentamientos, con cierto acompañamiento y consenso político.

Mediante un estudio que vincula la inscripción territorial con las políticas sociales como parte de un nuevo repertorio de acción de las clases populares, Merklen (1997) plantea que han existido dos momentos en la organización de los asentamientos. Uno ligado a la amplia participación de los habitantes del barrio, en donde las "organizaciones se construyeron sobre la idea de la autonomía respecto de la competencia político-partidaria" ( $p$. 6), como a-partidarios o a-políticos. Y el otro, hacia 1988, donde "con la llegada del peronismo al poder de la provincia de Buenos Aires se plantea un nuevo vínculo con los asentamientos, ya no conocido por el deseo de erradicarlos" (p. 7).

En este contexto, tal como señala el citado autor, se crea a nivel provincial el plan Pro-Tierra, que entre sus objetivos incluía la ayuda a la consolidación de las organizaciones comunitarias de los barrios y la venta de las tierras tomadas a sus ocupantes, y donde toman protagonismo, según Merklen (1997), algunas organizaciones de base territorial como la Federación de Tierra y Vivienda (FTV) y la Corriente Clasista y Combativa (CCC) en la Matanza. Al mismo tiempo, en Lomas de Zamora, se lanza "Casas con Tierras" con objetivos similares al plan provincial. Cabe añadir que, por esos años, otras organizaciones sociales y ONGs comienzan a adquirir mayor protagonismo, como es el caso de Caritas, desde donde surgió la organización Madre Tierra que actuó en la zona noroeste del Conurbano sobre las áreas de influencia del obispado de Merlo-Morón.

A partir del accionar de las organizaciones sociales y su conexión con la política partidaria, Cravino (2009) sostendrá que las ocupaciones de tierras se "institucionalizaron" como forma de acceso al suelo urbano. En este sentido, ya entonces se pueden ver las fuertes vinculaciones entre diferentes actores de anclaje territorial y la construcción de nuevos asentamientos en sus zonas de influencia, e incluso más allá, la conformación de nuevos territorios de asentamientos, en donde las organizaciones políticas que participarán y la venia de los gobiernos municipales serán determinantes.

Así, desde esa "institucionalización" se comienzan a ocupar predios pertenecientes, además de a la Iglesia Católica, al Estado, a los sindicatos de trabajadores, como asimismo predios privados abandonados o degradados ambientalmente.

\section{Tercer momento. El descenso de las ocupaciones, en la organización peronista por la subsistencia} Desde mediados de los 90 y hasta el 2003, debido a la recesión económica, según Cravino (2009), no hubo un crecimiento significativo de los asentamientos, sobre todo porque no había expectativas de mejora de los ingresos, y desde las agencias gubernamentales no existía una recepción de las demandas de la población en cuanto a vivienda. 


\begin{tabular}{|c|c|c|c|c|c|c|}
\hline GBA & Municipios & 2015 al 2011 & $\begin{array}{c}\text { Entre } 2010 \text { y } \\
2003\end{array}$ & $\begin{array}{c}\text { Anterior al } \\
2003\end{array}$ & Total & $\begin{array}{l}\text { Subtotal del } \\
2015 \text { al } 2003\end{array}$ \\
\hline 1er & Avellaneda & 0 & 0 & 5 & 5 & 0 \\
\hline \multirow[t]{8}{*}{ Cordón } & San Martín & 0 & 0 & 4 & 4 & 0 \\
\hline & San Isidro & 0 & 0 & 1 & 1 & 0 \\
\hline & Morón & 0 & 0 & 1 & 1 & 0 \\
\hline & Ituzáingo & 0 & 0 & 6 & 6 & 0 \\
\hline & Hurlingham & 0 & 0 & 18 & 18 & 0 \\
\hline & Vicente López & 0 & 0 & 0 & 0 & 0 \\
\hline & Lanús & 0 & 0 & 0 & 0 & 0 \\
\hline & Tres de Febrero & 0 & 0 & 0 & 0 & 0 \\
\hline 2 do & Moreno & 28 & 16 & 13 & 57 & 44 \\
\hline \multirow[t]{14}{*}{ Cordón } & Florencio Varela & 20 & 11 & 25 & 56 & 31 \\
\hline & José C. Paz & 16 & 10 & 12 & 38 & 26 \\
\hline & La Matanza & 9 & 28 & 23 & 60 & 37 \\
\hline & $\begin{array}{c}\text { Almirante } \\
\text { Brown }\end{array}$ & 5 & 21 & 25 & 51 & 26 \\
\hline & Merlo & 3 & 6 & 12 & 21 & 9 \\
\hline & $\begin{array}{l}\text { Esteban } \\
\text { Echeverría }\end{array}$ & 2 & 7 & 12 & 21 & 9 \\
\hline & $\begin{array}{c}\text { Malvinas } \\
\text { Argentinas }\end{array}$ & 0 & 15 & 12 & 27 & 15 \\
\hline & Tigre & 0 & 3 & 13 & 16 & 3 \\
\hline & $\begin{array}{l}\text { Lomas de } \\
\text { Zamora }\end{array}$ & 0 & 9 & 28 & 37 & 9 \\
\hline & Ezeiza & 0 & 0 & 0 & 0 & 0 \\
\hline & Berazategui & 0 & 1 & 6 & 7 & 1 \\
\hline & Quilmes & 0 & 2 & 0 & 2 & 2 \\
\hline & San Miguel & 0 & 3 & 5 & 8 & 3 \\
\hline & San Fernando & 0 & 0 & 2 & 2 & 0 \\
\hline
\end{tabular}




\begin{tabular}{|c|c|c|c|c|c|c|}
\hline 3er & Pilar & 0 & 21 & 1 & 22 & 21 \\
\hline \multirow[t]{5}{*}{ Cordón } & $\begin{array}{l}\text { General } \\
\text { Rodríguez }\end{array}$ & 0 & 2 & 0 & 2 & 2 \\
\hline & $\begin{array}{l}\text { Belén de } \\
\text { Escobar }\end{array}$ & 0 & 7 & 14 & 21 & 7 \\
\hline & Marcos Paz & 0 & 1 & 0 & 1 & 1 \\
\hline & San Vicente & 0 & 9 & 0 & 9 & 9 \\
\hline & Cañuelas & 0 & 0 & 1 & 1 & 0 \\
\hline \multicolumn{2}{|c|}{ Totales } & 83 & 172 & 255 & 510 & 255 \\
\hline \multirow{2}{*}{\multicolumn{2}{|c|}{ Primeros 5 por período }} & 78 & 86 & 98 & 262 & 164 \\
\hline & & $-94 \%$ & $-50 \%$ & $-38 \%$ & $-51 \%$ & $-64 \%$ \\
\hline
\end{tabular}

Tabla 1. Tomas totales por municipios del GBA, según años. Fuente: Elaboración del autor en base al Registro Provincial de Villas y Asentamientos por Ley de Acceso Justo al Hábitat 14.449 y Google Earth.

En igual dirección, Merklen (1997) señalará el papel del peronismo y su organización en relación a la contención social de los sectores más empobrecidos de la sociedad, que se concentraban, a través del asistencialismo, en el sostenimiento de un modelo en descomposición basado en la paridad monetaria con el dólar y la liberalización económica, y que traía aparejada la desindustrialización y, en consecuencia, el aumento del desempleo. Las políticas de distribución de mercaderías alimentarias básicas a través de "las manzaneras" del barrio garantizan la subsistencia de la población, pero lejos quedan las acciones asociadas a las reivindicaciones de acceso al suelo o la vivienda.

Cuarto momento. Nuevas tomas y viviendas de interés social en algunos municipios de la segunda y tercer corona del GBA Desde 2003, período que se revisará más adelante, volvió a crecer la cantidad de ocupaciones, fundamentalmente por mejores expectativas de empleo e ingresos crecientes, lo que se vio acompañado por la migración de población desde países limítrofes y provincias del norte. Diversas políticas en materia de mejora y construcción de vivienda se adoptaron por estos años, pero no se dio una transformación "de fondo" que permitiera el acceso a suelo urbano por vías formales a las clases de menores ingresos.

En este contexto, se observaba un nuevo movimiento de ocupaciones de tierra, al mismo tiempo que se comenzaban a ofertar viviendas de interés social en cantidades nunca dadas; "de esta forma, algunas de las ocupaciones fueron, en realidad, un medio para presionar por el acceso a dichas viviendas y no una intención de ocuparlas en sí" (Cravino, 2009, p. 39).

\section{Configuraciones actuales de los territorios de asentamientos}

Desde el año 2003 hasta el 2015 las tomas en el borde metropolitano se incrementaron de modo exponencial dada la mejora de las expectativas económicas y de empleo, lo que además traccionó un flujo migratorio de población de clase social baja de países de la región (Cravino, 2016), sobre todo de Paraguay, y en menor medida, de Perú y Bolivia, así como población de argentinos, desplazados desde las áreas centrales de la ciudad (algunos de los cuales ya radicaban en villas) o provenientes de otras provincias del país. En este período se tuvo una política permisiva respecto a las nuevas tomas, en aras de urbanizar los barrios y evitar desalojos violentos. En otro plano, se aprobó una amplia gama de programas y normativas que buscaron resolver la problemática de acceso al suelo y, más ampliamente, a la ciudad $\mathbf{5}$.

Como puede verse en el siguiente cuadro (Tabla 1), que considera los asentamientos en el GBA, existen grandes diferencias entre los distritos en cuanto a la cantidad de asentamientos que poseían hasta 2015, así como los barrios que se dieron por nuevas tomas en distintos períodos. Aquí se analizan las tomas en los siguientes cortes: antes de 2003, entre 2003 y 2010 y, finalmente, de 2011 a 2015.

Si se consideran las tomas de los últimos años (2011-2015), es posible observar que los primeros cinco municipios concentran el 94\% de las totales, siendo los más relevantes Moreno, Florencio Varela, José C Paz, La Matanza y Almirante Brown (en ese orden), todos municipios de la segunda corona; mientras que 


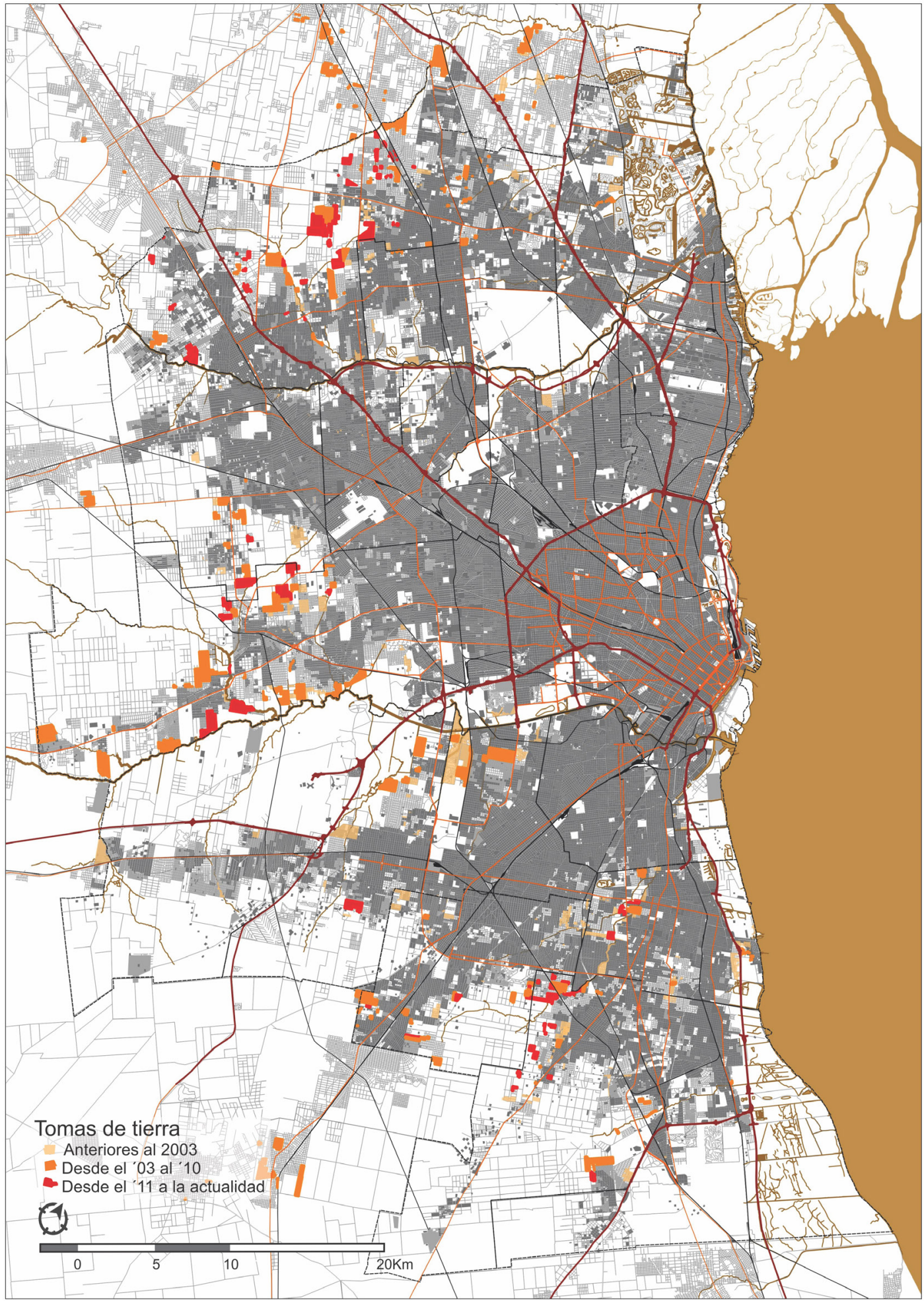

Figura 2. Actuales asentamientos según años de ocupación. Fuente: Elaboración del autor a partir del Registro Provincial de Villas y Asentamientos por Ley de Acceso Justo al Hábitat 14.449, y relevamiento través de Google Earth. 


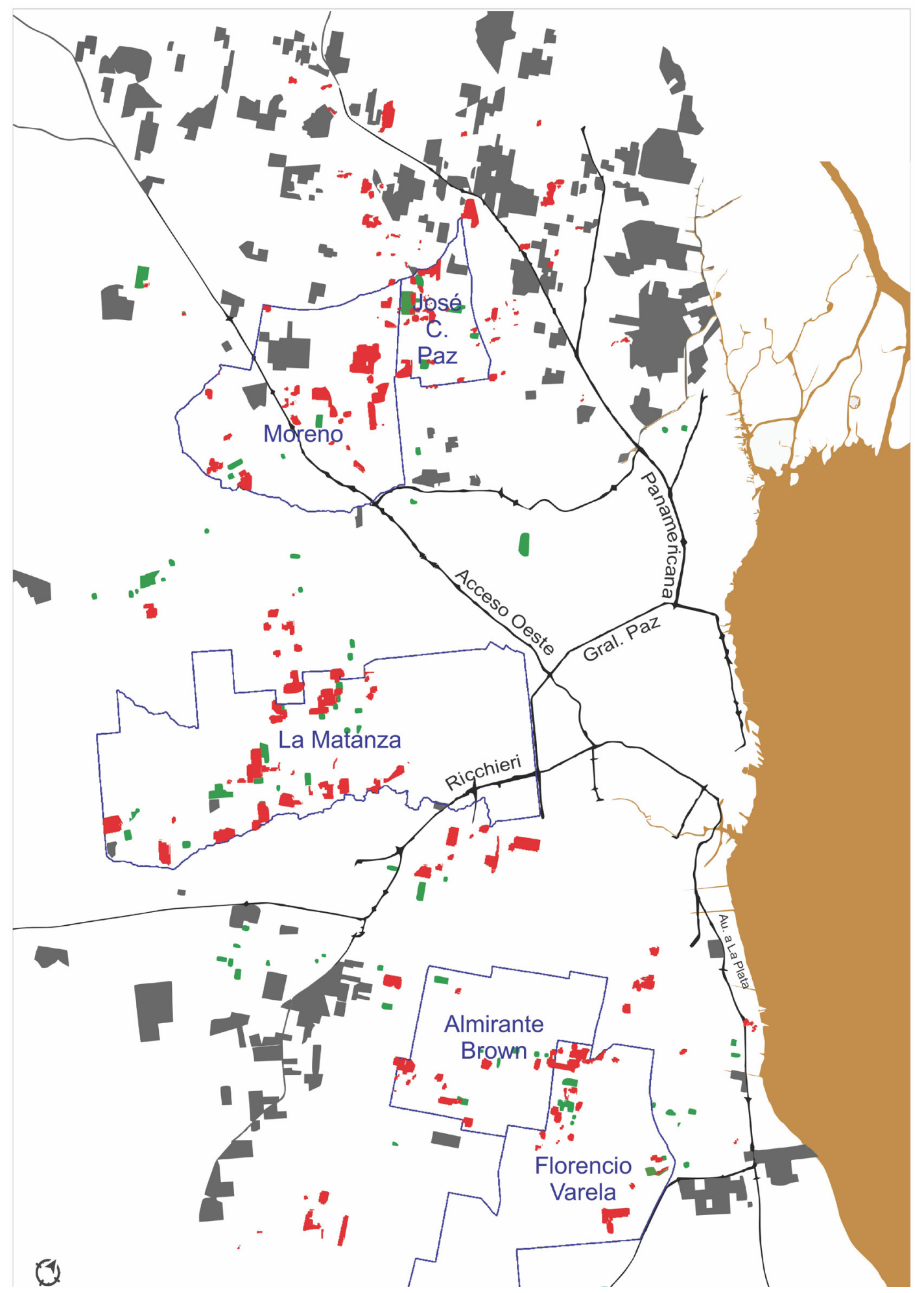


en la primera y tercera corona no se han registrado nuevos barrios. Si se observa el corte temporal 2003 a 2010, se puede identificar que, por amplio margen, las tomas se concentraron en tres de los municipios antes mencionados, además de Malvinas Argentinas y Pilar.

A partir de lo anterior, se advierte que los asentamientos, como problema, no constituyen un tema del GBA en su totalidad, y que tampoco se concentran especialmente en los municipios de la segunda corona como algunos autores afirman (Apaolaza y Venturini, 2018). En efecto, históricamente, este fenómeno se ha dado en áreas muy puntuales y, en los últimos años, se ha concentrado con fuerza en cinco municipios repartidos entre la zona sur, la noroeste y la oeste. En el siguiente mapa (Figura 2) se puede apreciar lo analizado en el cuadro anterior.

Entendiendo que los municipios parecen ser el ámbito natural donde los dirigentes o militantes barriales son contenidos políticamente (Cravino, 2009), en algunos, como La Matanza, es importante considerar el papel que jugaron organizaciones de base territorial, como la Federación de Tierra y Vivienda y la Corriente Clasista y Combativa o el Frente de Organizaciones en Lucha (FOL), como movimientos vinculados a los procesos de tomas de tierra, desde el acompañamiento a las familias necesitadas. En otros municipios, como en Moreno, también es destacable el papel de la asociación civil Madre Tierra junto al del Instituto Autárquico Municipal (IDUAR), que han tenido por política no acompañar los pedidos de desalojo de las tomas y llevar adelante un proceso de integración socio-urbana y acompañamiento. En otros municipios, movimientos sociales y organizaciones han tenido alguna participación, como el Movimiento Evita, Barrios de Pie, la CETEP, entre otros, los que se han visto mejor contenidos en determinadas administraciones. De este modo, en los últimos años ha habido diferentes gobiernos locales -con continuidad en las gestiones- que han sido más o menos permeables a los procesos de extensión de los asentamientos, ya sea por acción u omisión. Además, dichos gobiernos no han podido romper con una dinámica de segregación que ha ido mucho más allá de las políticas que hubieran podido implementar.

Las transformaciones de escala metropolitana que se dieron desde la reactivación económica del año 2003 operaron cambios radicales en cuanto a la producción de vivienda para todas las clases sociales. Por un lado, estuvieron las administraciones que se encontraban servidas por las autopistas y poseían un suelo más codiciado por el mercado inmobiliario para el desarrollo de grandes emprendimientos, como parques industriales, grandes superficies comerciales o barrios y urbanizaciones cerradas. Este proceso fue estudiado tanto desde el punto de vista morfológico (Colella, 2019), como en relación al mercado inmobiliario (De Mattos, 2016) o al proceso de producción y consumo de la urbanización (Pírez, 2016). Por otro lado, existieron administraciones que se centraron en receptar planes y programas provinciales y nacionales con la radicación de barrios de viviendas de interés social. Según Aramburu y Chiara (2016), en el período de 2004 a 2014, en los municipios del GBA la construcción de viviendas del Programa Federal fue en un 50\% del total, implementada sobre tres de los veinticuatro municipios de la primera y segunda corona: José $C$. Paz (con 5.316 viviendas), Florencio Varela (4.540) y La Matanza (3.814), seguidos luego por Moreno (3.218) y Esteban Echeverría (2.252). Considerando que el programa suponía la asignación de viviendas a los municipios que ponían el suelo a disposición, según sus posibilidades, las administraciones beneficiadas fueron las que poseían grandes extensiones de tierras en áreas con precios relativos bajos en zonas de borde. El siguiente esquema (Figura 3) intenta poner en relación los múltiples factores que han operado en la determinación de las áreas ocupadas por nuevos asentamientos y han definido los territorios. Puede observarse la preeminencia de tomas de tierra en los municipios señalados, la vinculación estrecha con los barrios de viviendas de interés social y, en contraposición, los barrios cerrados conectados a las autopistas.

\section{V.CONCLUSIONES}

El presente artículo ha buscado dar cuenta de lo que se ha postulado como "territorios de asentamientos", considerando desde las primeras tomas en el conurbano (y otros antecedentes) hasta las más recientes. Desde este enfoque, se pudo visibilizar las áreas que se ocuparon, quiénes participaron del fenómeno, cómo lo hicieron, y cómo esto fue percibido y estudiado a través del tiempo. Así, se pudo explicitar algunas relaciones y dar cuenta de sus especificidades, concretamente entre los actores involucrados en el proceso de tomas de tierra y el tipo de suelo ocupado, su localización espacial y temporal, su dispersión o concentración a nivel metropolitano.

Desde la dimensión metropolitana, diversos factores explican la extensión de los asentamientos y su localización, tal como se pudo evidenciar, de los cuales algunos son constantes mientras que otros han cambiado. En primer lugar, se puede afirmar que las especificidades físicas del suelo, su tenencia jurídica y la zonificación que le corresponde ocupa un lugar relevante. Con muy pocas excepciones, siempre se ha tratado de zonas de borde urbano, de suelo degradado, con cavas o inundable, generalmente de tenencia irregular, y en zonificación de tipo rural o complementaria contigua a un área urbana. En el mismo sentido, el costo del suelo tuvo un papel fundamental, pero ha tomado mayor relevancia en los últimos años, sobre todo a partir de una creciente segregación residencial y la presión predominante del mercado inmobiliario en el suelo de la periferia. Pero más allá de los diferentes factores mencionados, los actores que se han construido en torno al proceso de asentamientos jugaron un papel fundamental.

Las políticas de hábitat y vivienda de la que fueron objeto o impulsaron los gobiernos locales, que entre 2003 y 2015 se profundizaron y se sumaron a los programas nacionales y provinciales, tuvieron un impacto diferencial entre las administraciones que optaron por incorporarlas y las que no, resultando en una recepción de población por parte de las primeras 
que desbordó las capacidades de gestión que poseían. Las áreas de influencia o divisiones jurídicas o administrativas, ya sea de gobiernos locales, grupos religiosos, organizaciones comunitarias o políticas y cooperativas, también ocupan un papel relevante en la determinación de las áreas a ocuparse. Los gobiernos locales siempre han tenido un papel fundamental en la materia, tal como ha quedado expuesto en el desarrollo de este artículo, pero el papel de las organizaciones religiosas, como la Católica y, más recientemente, las Evangélicas no es menor. Por otra parte, en el período que aquí fue tomado como el cuarto momento, las organizaciones sociales, políticas y cooperativas han tenido mucha influencia a partir de una participación activa y con posibilidades de financiamiento para diferentes proyectos. En esa materia, las vinculaciones entre estos actores de diferente pertenencia constituyen entramados con anclaje territorial capaces de tener un peso predominante a nivel metropolitano sobre las ocupaciones de tierras, así como también en las posibilidades de llevar adelante programas de integración socio-urbanos y de regularización.

Finalmente, si bien aún queda mucho por trabajar, entender mejor estos territorios desde la perspectiva propuesta puede ser una herramienta fundamental para definir o redefinir políticas territoriales que orienten los procesos desde el acompañamiento a los actores y redes de actores que tienen relevancia a escala metropolitana, así como para contribuir al conocimiento de los asentamientos como objeto de estudio. En esa línea, además sugiere un modo de explorar y dar cuenta de ciertas especificidades de los diferentes tipos de hábitat informal que han sido, hasta ahora, poco exploradas.

\section{REFERENCIAS BIBLIOGRÁFICAS}

Amin, A. (2005). Regiones sin fronteras: hacia una nueva política de lugar. Revista Ekonomiaz, 58(1), 76-96.

Apaolaza, R. y Venturini, J. (2018). Expansión urbana, desplazamiento y periferización popular. Breve diagnóstico de la evolución de los nuevos asentamientos informales periféricos de la Región Metropolitana de Buenos Aires. En Seminario Latinoamericano "Teoría y Política sobre Asentamientos Populares". Universidad Nacional de General Sarmiento, Los Polvorines, Buenos Aires.

Aramburu, F. y Chiara, C. (2016). Los planes federales de vivienda en el área metropolitana de Buenos Aires. En IX Jornadas de Sociología, Universidad Nacional de La Plata, Ensenada, Argentina. Recuperado de http://www. memoria.fahce.unlp.edu.ar/trab_eventos/ev.9056/ev.9056.pdf

Chiara, M. y Catenazzi, A. (2009). La participación en la gestión: alcances y límites en su institucionalización. En M. Chiara y M. Di Virgilio (Coords.), Gestión de la Política Social. Conceptos y Herramientas (pp. 201-214). Buenos Aires, Argentina: Prometeo.

Clichevsky, N. (1991). Tierra fiscal y regularización urbana. Informe de Investigación. Buenos Aires: IDRC-IIED-ALCONICET.

Clichevsky, N. (2003). Pobreza y acceso al suelo urbano. Algunos interrogantes sobre las políticas de regularización en América Latina. Serie Medio Ambiente y Desarrollo. CEPAL, Naciones Unidas (75).

Colella, V. (2019). Persistencias, transformaciones y resistencias urbanas en la costa norte del AMBA. Tesis doctoral FADU - UBA.
Cravino, M. (2001). La propiedad de la tierra como un proceso. Estudio comparativo de casos en ocupaciones de tierras en el Área Metropolitana de Buenos Aires. En Land Tenure Issues in Latin Ámerica. Slas 2001, Conference. Birmingham.

Cravino, M. (2009). La metamorfosis de la ciudad informal en el Área Metropolitana de Buenos Aires. Revista Lider, 15(11), 31-55.

Cravino, M. (2016). Desigualdad urbana, inseguridad y vida cotidiana en asentamientos informales del Área Metropolitana de Buenos Aires. Etnografías Contemporáneas, 2(3), 56-83. Recuperado de http://www.unsam.edu.ar/ojs/ index.php/etnocontemp/article/view/169

Cravino, M. (2018). Evolución cuantitativa y transformaciones cualitativas de los asentamientos populares del Área Metropolitana de Buenos Aires (19802015). En M. Cravino (Org.), La ciudad (re)negada. Aproximaciones al estudio de asentamientos populares (pp. 67-102). Los Polvorines, Argentina: Universidad Nacional de General, Sarmiento Ediciones.

De Mattos, C. (2016). Financiarización, valorización inmobiliaria del capital y mercantilización de la metamorfosis urbana. Sociologías, (42), 24-52.

Di Virgilio, M., Arqueros Mejica, M., Guevara, T. (2012). Estrategias de acceso al suelo y a la vivienda en barrios populares del Área Metropolitana de Buenos Aires. Revista Brasileira de Estudos Urbanos e Regionais, 14(1), 29-49.

Governa, F. (2007). Las infraestructuras de transporte concebidas como obras territoriales. Papers: Regió Metropolitana de Barcelona: Territori, estratègies, planejament, (44), 20-31.

Hardoy, J.y Satterwhite, D. (1987). La ciudad legaly la ciudad ilegal. Buenos Aires, Argentina: GEL.

Izaguirre, I. y Aristizabal, Z. (1988). Las tomas de tierra en la zona sur del Gran Buenos Aires. Una experiencia de poder popular. Buenos Aires, Argentina: CEAL.

Merklen, D. (1997). Organización Comunitaria y Práctica Política. Las Ocupaciones de Tierras en el conurbano de Buenos Aires. Revista Nueva Sociedad, 149, 162-177.

Merlinsky, G. (2017). Cartografias do conflito ambiental na Argentina. Notas teórico-metodológicas. 1 Acta sociológica, (73), 221-246.

Pacheco, J. (2018). Sobrepoblación relativa, acción política y dictadura militar en la Ciudad de Buenos Aires, 1976-1983: las organizaciones villeras frente a la política estatal de erradicación masiva. URBS. Revista de Estudios Urbanos y Ciencias Sociales, 8(2), 63-71.

Pírez, P. (2016). Las heterogéneas formas de producción y consumo de la urbanización latinoamericana. IGG, UBA. Quid 16. 6, 131-167.

Pradilla, E. (1982). Autoconstrucción, explotación de la fuerza de trabajo y políticas del Estado en América Latina". En E. Pradilla (comp.), Ensayos sobre el Problema de la Vivienda en América Latina, Universidad Autónoma metropolitana-Xochimilco, México DF, México.

Thoenig, J.C. (1997). Política pública y acción pública. Gestión y Política Pública, $1,19-37$.

Turner, John (1977). Todo el poder a los usuarios. Blume Ediciones, Madrid.

Ugalde V. (2014). Sociología de la acción pública. Una mirada a nuevos enfoques. Traducido de Lascoumes, P. y Le Galès, P. (1984), Sociologie de l'action publique. Desafíos, 27(2), 325-330 


\section{THE SETTLEMENT TERRITORIES ON THE METROPOLITAN FRINGES OF BUENOS AIRES, FROM 1980 TO THE PRESENT DAY}

\author{
LUCAS JORDÁN DOMBROSKI
}

\section{INTRODUCTION}

The informal habitat is a relevant issue in Latin America, and a wide range of debates has arisen around this, especially since the 1970 s, that try to provide answers to the phenomenon at a regional scale (Turner, 1977, Padrilla, 1982, Hardoy \& Satterthwaite, 1987, Clichevsky, 2001). More recently, studies have focused on case analysis, where they try to understand the specificities of the phenomenon for each site. This, given that the issue is substantially different among the largest cities on the subcontinent. While for cities like Mexico City, Sao Paulo, Rio de Janeiro, Lima, Caracas or Bogota, informality represents between 30 and 60 percent, in others like Santiago or Montevideo, it is around 10 percent (Clichevsky, 1991). The same applies for Greater Buenos Aires 3 (GBA), with a total of one and a half million inhabitants living in more than a thousand neighborhoods (Cravino, 2018). The specificity of the processes is also different in environmental, social and political terms. In the GBA, the "settlements" 4 or "land occupation" have been subject of academic studies, press columns, debates about government administration, and policies since the end of the last century, when it was shown that this was a phenomenon that had come to stay, and was very different from the so-called "slums" and other informal habitat processes. Their location and the players involved in the process, have also been an important issue and have been the subject of different studies. Unlike the slums, which started to emerge in the 1930s and were concentrated in the city of Buenos Aires and the districts in the first ring; the settlements began to appear at the beginning of the 80 's and have been built on the fringes of the urbanized area. The specialized literature (Izaguirre \& Aristizabal, 1988; Merklen, 1997; Di Virgilio, 2012; Cravino, 2018) for this phenomenon considers, as an important matter, their location "in districts of the second ring" (Di Virgilio et al., 2012:32), in general, in unconsolidated areas "where most are located on private land, (...) on sites that, in general, were landfills, brushlands, or floodable areas, which is why the owners did not have any interest or possibility to exploit them economically or would suffer regulatory restrictions for this (Cravino 2001: 8)". In addition, over time, political and community organizations, organized neighbors, public servants, and other players who have taken part in the land occupation were defined as different to those from the slums, and they have been changing as time has gone by, growing as such around the settlement process. As will be seen throughout the article, many works explain the reason behind the emergence and growth of the occupations, the players involved, the physical specificities of the neighborhoods, or their localization. However, some of the relationships between these specificities over time had limited research. The goal of this work is to reconstruct the interpretation of the land occupation process in GBA between 1980 and the present day, related to the players involved and the specificities of the land that has been occupied, in order to understand the sites in play and their purpose in the Metropolitan area. This implies reviewing different material, from specialized texts to primary qualitative sources, along with quantitative data and plans. From the viewpoint of public action, with the analysis of the actions of several players that take place around the extension process of the settlements over time, it is understood that this interpretation brings to light the material and social particularities, and is key to distinguish the process and to design more effective public policies.

\section{THEORETICAL FRAMEWORK}

Different areas and perspectives of analysis regarding the study of the players and the territory itself contribute to this work. From there, and regarding the works that have addressed public policies, it is deemed necessary to deconstruct the idea of the State, that is assumed as monolithic in a hierarchical, rational and static direction of the policies (Lascoumes; Le Galés, 1984), that are created around the informal habitat, and consider instead, the interaction of the players in terms of public authorities and social players. This as the implementation and redefinition of the habitat policies are played out onsite, where the players become so from their participation (Chiara \& Catenazzi, 2009). The latter leads to talking about public action, not just regarding the policies in their preparation and implementation, but in a more general sphere that implies considering urban transformations in general, and that allows visualizing more accurately, the actions of public authorities and considering, in a different way, the social players in their capacity to impact said transformations.

Now, what is understood by public action? In theoretical terms, and for the study of settlements, it must be understood as Thoenig (1997:30) outlines, where the public power does not have a monopoly over politics, and transformations, in this

3 Here, Greater Buenos Aires is considered as comprising the Autonomous City and 30 districts that surround it, successively forming three rings or "coronas", from the center to the outskirts.

4 There is agreement among researchers who are specialized in the study of the informal habitat in the GBA regarding the denomination of "land occupation" and "settlements (plainly speaking)" as equivalent. The denomination "informal settlements" or "popular settlements" refers to a more general process that includes, for example, the "slums". 
case the size of the settlements, "takes place in an 'arena', where different logics and values, very diverse modalities, circumstances and planned interventions, technical rationality and political elections, scientific expertise and militant commitments, scheduling and concertation, coexist". This means considering the study of the players and territorial transformations from that 'arena', which some authors operationalize around the identification of different 'moments' (Merlinsky, 2017) of interaction or turning points in a process. The considerations of the territory are played out on a different plane, but here interact with that of public action as a notion, as the territory is understood as a "dynamic and active area, a social structure that arises from the interaction of the subjects and the specific and physical material and immaterial characteristics of the different spaces" (Governa, 2007:23) which is why, apart from being a specific area comprising extensions, limits and obstacles, it also consists of flows and nodes that act as networks of relations and connections of the players (Amin, 2005). Starting from this basis, this work focuses on some of the territorial specificities that may be of interest from a metropolitan scale.

\section{METHODOLOGY}

The settlement territories, as an object of study, and in the terms that are of interest here, comprise the players involved from the point of view of public action - and the specificities of the places where the neighborhoods are located, both spatially and temporally.

The study technique consisted of historic review about the interpretations of the land occupation process that took place between the 1980s and the start of this century. For more recent years, the technique was based on building a metropolitanscale map with the geographic and temporary location of the neighborhoods and the identification of the different players involved.

Specialized texts were used, paying special attention to the valuations of the authors about the features of the occupied areas, the localization of the occupation, the players involved, their means of organization and their relations with other players. To rebuild more recent processes, data from web platforms was used (Provincial Record of Slums and Settlements through the Fair Access to Habitat Act, Law 14.449, the National Working-Class Neighborhood Record and Google Earth). In addition, at a different level of approach, the case studies that resulted from the doctoral work made by the author were relevant, from the contribution of information about the territories both in their material and immaterial specificities. Semi-structured interviews with key players (a total of 15 made with municipal workers, standout neighbors and organization members), the preparation of targeted maps and field work carried out from the study of neighborhoods of local districts with the most occupations (Moreno, José C. Paz and La Matanza), helped to understand why these areas were occupied and how, the players that took part, the policies that were applied and the strategies and resources that were deployed. From the reconstruction of the occupation process, different "moments" could be identified regarding the articulation of the players involved and the areas that were occupied. Thus, this is about territorial specificities over time, which will be brought to light later in this article.

\section{RESULTS}

The results are presented in two parts. The first is linked to the analysis of the literature over a period of time between the 1980's and 2003. The second is from 2003 onwards, where progress is made about the quantitative and qualitative data of the phenomenon.

\section{Construction, from the literature, of the settlement territo- ries in recent decades}

Before moving onto the extensive path that settlements have taken to the present day, it is necessary to look back at their origin. This as, until the 70's, there were less restrictive possibilities of accessing land and housing for the working class compared to today, related to State policies and market dynamics. The role of the measure to freeze rentals and "cheap parceling" (without requiring utilities) stands out (Cravino, 2001; Di Virgilio et al., 2012) This ended during the civilian-military dictatorship, with the sanction of the Urban Locations Law $N^{\circ} 21342$ from 1976 and the Decree in Law of Land Use and Territorial Regulations N8912 from 1977. As part of the series of measures, it is also necessary to consider the sanction of the Municipal Planning Code of Buenos Aires and the eviction of population through the eradication of the slums (Pacheco, 2018), or due to demolitions to build highways (Izaguirre \& Aristizabal, 1988; Cravino, 2001).

By analyzing the specialized literature, four moments in the settlement territory construction process can be seen.

First moment. The occupations in the southern area and the church organization

Around the start of the 80 's, during the decline of the civilianmilitary dictatorship, settlements arose in the southern part of Greater Buenos Aires as a new phenomenon, especially occupations of large empty lots. This was related to the work of church-based groups which, to a great extent, due to the overriding political outlawing of those years, had become a key player in containing the impoverished and displaced population. Church organizations, as the years went by, were building their influence by accompanying the occupations that took place, as Cravino (2001:10) stated, from the south, "towards the west and, to a lesser extent, the north, where the high-income sectors were concentrated".

One of the first works that refers to the settlement phenomenon 
is that of Izaguirre \& Aristizabal (1998), which talks about the collective and organized occupation of the land, considering the role of the working classes and their transformation in the context of the dictatorship and the return to democracy. It is from this moment that a direct link is suggested between the generation of major infrastructure works for the GBA and the localization of the land occupations. Thus, the projection of a partial construction and setup of the Green Belt and the Buen Ayre Highway were set up as projects that marked off the areas that were meant to be free of settlements from those that could be susceptible to new occupations. The authors understand that, therefore, there was a "series" of measures that had different impacts on the affected population groups, and point out, in this regard, a very clear intentionality: "pushing the urban poor towards the outskirts of parts of Greater Buenos Aires, beyond the so-called "green belt" clean corridor, a future green reserve area that, under the ideological robes of "clean air", facilitated the strategic control of the poorest sectors...", "spreading them to these areas in a far wider radius, which limited their threatening concentration near the seat of the groups of power" (Izaguirre \& Aristizabal, 1988: 7) The following map (Figure 1), built from the data collected by Aristizabal, shows the settlements that arose from the occupations in 1981 and those which took place later, now in democracy between 1985 and 1986. Although the records from that period are not that accurate and are based on journaliststyle reports, the importance of the occupations is clearly seen along the line between the Almirante Brown and Quilmes districts, which share nine neighborhoods between them, on the banks of the Las Piedras and San Francisco streams, in the area of influence of the Diocese of Quilmes (six in ' 81 and three in '85). Later, in '86, three occupations were recorded in La Matanza, one in Merlo and one in Morón, but all smaller in terms of surface covered and number of families.

Figure 1. Land occupation from 1981 to 1985 . Source: Own preparation using the records of Izaguirre \& Aristizabal (1988)

\section{Second moment. Occupations in some districts of the second ring, from the "non-partisan" organizations to the links with Peronism.}

In the first years of democracy, during radicalism, "the state position had aimed at trying to avoid occupations, facing off with the occupant movements" Merklen (1997: 7). From the Peronist government, the relationship was presented in a different way, thus building very specific areas where settlements were developed, with a certain accompaniment and political consensus.

From a study which links territorial registration with social policies as part of a new repertoire of action of the working classes, Merklen (1997: 6) states that there have been two moments in the organization of the settlements. A first one linked to the broad participation of the neighborhood's inhabitants, where the "organizations were built upon the idea of autonomy regarding the political parties", as non-partisan or apolitical, and a second towards 1988, where "with the arrival of Peronism to power in the Province of Buenos Aires, a new link with the settlements is seen, no longer recognized by the desire to eradicate them". In this context, just as the author outlines, the Pro-Land plan is created at a provincial level, which among its goals included support to consolidate the community organizations of the neighborhoods and the sale of occupied land to its occupants. Here, leadership was taken, according to Merklen (1997), by some territorially-based organizations like the Land and Housing Federation (FTV) and the Classist and Combative Current (CCC) in La Matanza. At the same time, in Lomas de Zamora, "Houses with Land" is launched with similar goals to the provincial plan. Also, during those years, other social organizations and NGOs begin to have a more relevant role, like the case of Caritas, where the Mother Earth (Madre Tierra) organization emerged from, acting in the northeast area of the city on the areas of influence of the Bishopric of Merlo-Morón. From the actions of social organizations and their political connections, Cravino (2009) will state that the land occupations were "institutionalized" as a way to access urban land. In this sense, already by then the strong ties between different territorially-anchored players and the construction of new settlements in their areas of influence can be seen, but furthermore, the structuring of new settlement territories, where the political organizations that will take part and the agreement of municipal governments will be determining factors.

Thus, from this "institutionalization", lands begin to be occupied that belonged to, apart from the Catholic Church, the State, workers unions, and private abandoned or environmentally damaged lots.

\section{Third moment. The fall in occupations, in the Peronist organization due to subsistence}

Towards the mid 90's and up until 2003, Cravino (2009) states that, due to the economic recession, there has not been a significant growth of the settlements, especially because there were no expectations regarding salary increases, and there was no reception by government agencies of the population's demands regarding housing.

In this same sense, Merklen (1997) will point out the role of Peronism and its organization with regard to the social support of the most impoverished sectors of society, which was then focused on sustaining a model that was falling apart. The basic food supply distribution policies through the "manzaneras" (voluntary women from the neighborhoods), guaranteed the subsistence of the population, while aspects related to granting access to the land or housing fell out of the limelight.

Fourth moment. New occupations and social housing in some districts of the GBA's second and third ring

From 2003 onwards, a period that will be seen later, the number of occupations started to grow again, especially because of greater expectations of employment and growing income, 
accompanied by population migration from neighboring countries and northern provinces. Diverse policies related to retrofitting and the construction of housing accompanied this period, but a "real" transformation that would provide formal access to urban land for lower-income classes did not happen. In this context, a new land occupation movement was seen, at the same time that social housing of social interest began to be offered in numbers that never happened; "in this way, some of the occupations were, actually, a means to pressure access to this housing and not an intention to really occupy them" (Cravino 2009: 39).

\section{Current setup of the settlement territories}

From 2003 to 2015, the occupations on the Metropolitan outskirts grew exponentially as a result of economic and employment expectations, which also brought migration of a lower-class population from neighboring countries (Cravino, 2016), especially from Paraguay, and to a lesser extent, Peru and Bolivia. The Argentinian population who lived in slums, or who came from other provinces in the country, was also displaced from the central areas of the city. This period saw a permissive policy regarding new occupations from the aim to urbanize the neighborhoods and avoid violent evictions. Likewise, on another plane, a broad range of programs and regulations was approved that sought to resolve the issue of access to land and, more broadly, to the city 5 .

As can be seen in the following chart (Table 1), which considers the settlements in the GBA, there are large differences between districts regarding the number of settlements they had up to 2015 , as well as the neighborhoods that appeared from the new occupations in different periods. Here, the occupations are analyzed in sections: before 2003, between 2003 and 2010 and finally, from 2011 to 2015.

If the occupations of recent years (2011 to 2015) are considered, it is possible to see that the first five districts have $94 \%$ of the total, with the most relevant ones being Moreno, Florencio Varela, José C. Paz, La Matanza and Almirante Brown, in that order, all districts in the second ring, while the first and third rings have not seen new neighborhoods. If the 2003 to 2010 period is seen, it is possible to identify that, by a wide margin, the occupations are concentrated in three of the aforementioned districts and, also, in Malvinas Argentinas and Pilar.

Table $N^{\circ} 1$. Total occupations by districts of the GBA, by years. Source: Own preparation based on the Provincial Record of Slums and Settlements by the Fair Access to Habitat Act, 14.449 and Google Earth.

Starting from this, it can be said that the settlements as a problem are not an issue of the whole of the GBA, nor are they specifically concentrated in the second ring like some authors say (Apaolaza \& Venturini, 2018). But rather, they have historically emerged in very precise areas, and in recent years have been mainly concentrated in five districts spread over the south, northeast and west areas. By quickly checking the following map (Figure 2), what has been analyzed in the previous table can be seen.

Figure 2. Current settlements by years of occupation. Source: Own preparation based on the Provincial Record of Slums and Settlements by the Fair Access to Habitat Act, 14.449 and Google Earth

Understanding that the districts seem to be the natural environment where the neighborhood activists or leaders are politically contained (Cravino, 2009) in some, like La Matanza, it is important to consider the role that territorially-based organizations played, like the Land and Housing Federation and the Classist and Combative Current or the Organizations in Combat Front (FOL), as movements linked to land occupation processes, through accompanying families in need. In other districts, like Moreno, the role of the Mother Earth civil association stands out along with the Autonomous Municipal Institute (IDUAR), whose policy has been not supporting occupation eviction requests and promoting a socio-urban integration and accompaniment process. In other districts, social movements and organizations have been involved, like the Evita Movement, Neighborhoods Afoot (Barrios de Pie), CETEP, among others, which have been better contained in given government periods. In the sense of the latter, in recent years there have been different local governments - with continuity in the processes - that were more or less permeable to the settlement extension processes, be this by action or omission. In addition, these governments could not break a segregation dynamic that went beyond the policies they could implement.

The transformations on a Metropolitan scale that emerged from the economic reactivation of 2003 led to radical changes regarding housing production for all social classes. On one side, there were administrations that were served by the highways and had land that was greatly coveted by the property market to build large projects like industrial parks, large commercial areas or enclosed developments and neighborhoods. This process was studied both from the morphological point of view (Colella, 2019) and that of the property market (De Mattos, 2016), or that of the urbanization consumption and production process (Pirez, 2016). On the other hand, there were administrations that focused on receiving provincial and domestic plans and programs with the placement of social housing neighborhoods. According to Aramburu and Chiara (2016), in the 2004 to 2014 period, in the GBA's districts, the construction of housing of the Federal Program was 50\% of the total implementation in three of the twenty-four districts of the first and second ring. These 
were: José C. Paz (with 5,306 homes), Florencio Varela $(4,540)$ and La Matanza $(3,814)$, followed by Moreno $(3,218)$ and Esteban Echeverría $(2,252)$. Considering that the program involved the allocation of housing to districts that made land available, according to their possibilities, the benefitted administrations were those that had large expanses of land in areas with relatively low prices on the outskirts. The following diagram (Figure 3) tries to connect the multiple factors that have been involved in determining the areas occupied by new settlements and that have defined the territories. The dominance of land occupation in the aforementioned districts can be seen, as can the close ties with the social housing neighborhoods, and on the other hand, the enclosed neighborhoods linked to the highways.

Figure 3. Settlements, social housing and enclosed neighborhoods, from 2003 to the present. Source: Own preparation based on the Provincial Record of Slums and Settlements and Google Earth

\section{CONCLUSIONS}

This article sought to show what has been labeled as "settlement territories", considering the first occupations in the city and other background information, right up to the most recent ones. From this approach, it has been possible to shed light on the areas that were occupied, those who took part, how they did it, and how this was seen and studied over time. Thus, some relationships could be explained and their specificities revealed, along with the players who took part in the land occupation process, the type of land occupied, its spatial and temporal location, its spread or concentration at a Metropolitan level.

From the Metropolitan dimension, diverse factors explain the size of the settlements and their location, as could be seen. Some of these are constant, while others have changed. First, it can be confirmed that the physical specificities of the land, its legal ownership and the zoning it is part of, play an important role. With very few exceptions, these have always been on the outskirts, on spoiled land, with pits or floodable areas, generally with irregular ownership, and in rural or complementary-type zoning adjoining the urban area. In the same sense, the cost of the land played a key role, but has become more relevant in recent years, especially with a growing residential segregation and the overriding pressure of the property market on the land in the outskirts. But beyond the different factors mentioned, the players that have come to be through the settlements process played a key role.

The habitat and housing policies these were subject to or were implemented by local governments, which were developed further in the 2003 to 2015 period and were added to the national and provincial programs, had a differentiating impact between administrations that choose to include them and those that did not, resulting in a population reception by the former that exceeded the capacities they had to manage them. The areas of influence or legal or administrative divisions, be these of local governments, religious groups, community or political organizations and cooperatives, also play a relevant role in the determination of the areas being occupied. The local governments always had an essential role in the matter, as has been made evident in this article, but the role of religious organizations, like the Catholic church and more recently the Evangelists, is not a minor one. On the other hand, in the period considered here as the fourth moment, the social, political and cooperative organizations have had a great deal of influence from an active participation and with possibilities to finance different projects. In this matter, the links between these different players, who have different origins, make up networks that are linked to the territory, which are able to have an predominant role at a metropolitan level over the land occupations, as well as over the possibilities to carry out socio-urban integration and normalization programs. Finally, although there is still much work to be done, understanding these territories from the proposed perspective can be an essential tool to define or redefine territorial policies that guide the processes, by accompanying the players and networks of players that are important at a metropolitan scale, who also contribute to understanding the settlements as an object of study. In this sense, a way to explore and account for certain specificities of the different types of informal habitat, that have not been explored enough, is also suggested. 
101

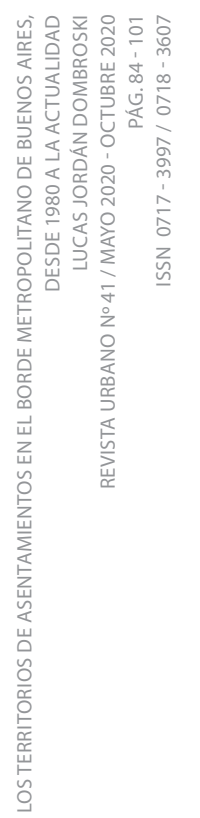

